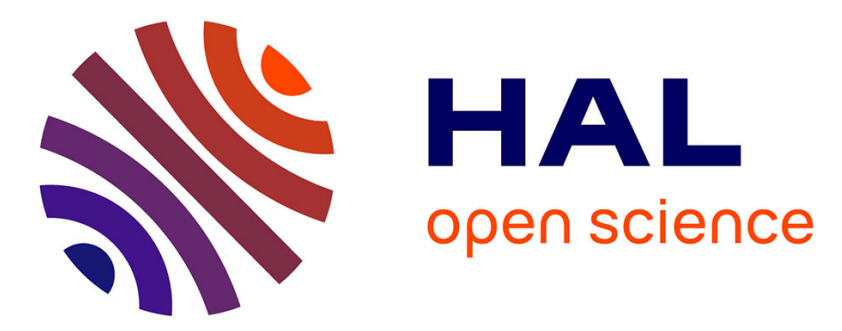

\title{
Mars heavy ion precipitating flux as measured by Mars Atmosphere and Volatile EvolutioN
}

François Leblanc, Ronan Modolo, Shannon Curry, Janet Luhmann, Rob Lillis, Jean-Yves Chaufray, Takuya Hara, Jim Mcfadden, Jasper Halekas, Frank Eparvier, et al.

\section{To cite this version:}

François Leblanc, Ronan Modolo, Shannon Curry, Janet Luhmann, Rob Lillis, et al.. Mars heavy ion precipitating flux as measured by Mars Atmosphere and Volatile EvolutioN. Geophysical Research Letters, 2015, 42 (21), pp.9135-9141. 10.1002/2015GL066170 . insu-01238144

\section{HAL Id: insu-01238144 https://hal-insu.archives-ouvertes.fr/insu-01238144}

Submitted on 3 Aug 2020

HAL is a multi-disciplinary open access archive for the deposit and dissemination of scientific research documents, whether they are published or not. The documents may come from teaching and research institutions in France or abroad, or from public or private research centers.
L'archive ouverte pluridisciplinaire HAL, est destinée au dépôt et à la diffusion de documents scientifiques de niveau recherche, publiés ou non, émanant des établissements d'enseignement et de recherche français ou étrangers, des laboratoires publics ou privés. 


\section{Geophysical Research Letters}

\section{RESEARCH LETTER}

10.1002/2015GL066170

\section{Special Section:}

First Results from the MAVEN

Mission to Mars

\section{Key Points:}

- Precipitation of heavy picked up ions occurs at Mars

- First estimate of the intensity of the precipitating flux on a statistically significant base

- First calculation of the sputtered atmosphere based on this measurement

Correspondence to:

F. Leblanc,

francois.leblanc@latmos.ipsl.fr

\section{Citation:}

Leblanc, F., et al. (2015), Mars heavy ion precipitating flux as measured by Mars Atmosphere and Volatile EvolutioN, Geophys. Res. Lett., 42, 9135-9141, doi:10.1002/2015GL066170.

Received 11 SEP 2015 Accepted 4 OCT 2015 Published online 5 NOV 2015

\section{Mars heavy ion precipitating flux as measured by Mars Atmosphere and Volatile EvolutioN}

\author{
F. Leblanc ${ }^{1}$, R. Modolo ${ }^{2}$, S. Curry ${ }^{3}$, J. Luhmann ${ }^{3}$, R. Lillis ${ }^{3}$, J. Y. Chaufray ${ }^{1}$, T. Hara ${ }^{3}$, J. McFadden ${ }^{3}$, \\ J. Halekas ${ }^{4}$, F. Eparvier ${ }^{5}$, D. Larson ${ }^{3}$, J. Connerney ${ }^{6}$, and B. Jakosky ${ }^{5}$ \\ ${ }^{1}$ LATMOS/CNRS, Université Pierre et Marie Curie, Paris, France, ${ }^{2}$ LATMOS, Université Versailles Saint Quentin, Guyancourt, \\ France, ${ }^{3} \mathrm{SSL}$, Berkeley, California, USA, ${ }^{4}$ Department of Physics and Astronomy, University of lowa, lowa City, lowa, USA, \\ ${ }^{5}$ LASP, Boulder, Colorado, USA, ${ }^{6}$ NASA Goddard Space Flight Center, Greenbelt, Maryland, USA
}

Abstract In the absence of an intrinsic dipole magnetic field, Mars' $\mathrm{O}^{+}$planetary ions are accelerated by the solar wind. Because of their large gyroradius, a population of these planetary ions can precipitate back into Mars' upper atmosphere with enough energy to eject neutrals into space via collision. This process, referred to as sputtering, may have been a dominant atmospheric loss process during earlier stages of our Sun. Yet until now, a limited number of observations have been possible; Analyzer of Space Plasmas and Energetic Atoms-3/Mars Express observed such a precipitation only during extreme conditions, suggesting that sputtering might be not as intense as theoretically predicted. Here we describe one example of precipitation of heavy ions during quiet solar conditions. Between November 2014 and April 2015, the average precipitating flux is significant and in agreement with predictions. From these measured precipitating fluxes, we estimate that a maximum of $1.0 \times 10^{24} \mathrm{O} / \mathrm{s}$ could have been lost due to sputtering.

\section{Introduction}

Luhmann and Kozyra [1991] suggested that heavy planetary ions (essentially $\mathrm{O}^{+}$, because $\mathrm{O}$ is the most abundant heavy neutral species in Mars' exosphere) are accelerated by the solar wind convective electric field and might reimpact Mars' atmosphere with energy up to few keVs. When these precipitating particles have energy from tens of eV up to $10 \mathrm{keV}$, they can induce a cascade of collisions in Mars' upper atmosphere, leading to the ejection of atmospheric particles with enough energy to exceed the escape velocity at Mars [Johnson et al., 2000]. This process, named sputtering, was suggested to potentially lead to significant atmospheric loss in its early history [Luhmann et al., 1992]. Measuring current-day sputtered ejecta with present measurement techniques is difficult because of the low energy (few eVs) and typical density of these sputtered particles with respect to other processes like photochemical escape [Lillis et al., 2015]. One of the objectives of the Mars Atmosphere and Volatile EvolutioN (MAVEN) is to determine if sputtering could have induced significant atmospheric loss over its history. As a first step, we focus on heavy ion precipitation, the primary source of sputtering.

Since the pioneering study by Luhmann et al. [1992], several successive works expanded on the original description of the precipitating heavy pickup ion fluxes into Mars' atmosphere (see Lillis et al. [2015] for references). These studies suggested a significant dependency of the flux intensity with respect to the orientation of the convective electric field, essentially determined by the interplanetary magnetic field (IMF) orientation and the solar wind conditions [Wang et al., 2014, 2015; Li et al., 2011]. Other works explored the dependency of precipitation flux intensity on the solar UV/EUV flux intensity [Chaufray et al., 2007] and also with respect to Mars' crustal field locations [Wang et al., 2014, 2015; Li et al., 2011]. Heavy ion precipitation is therefore expected to be highly variable on timescales typical of the IMF orientation variability with complex, highly nonuniform spatial patterns [Wang et al., 2014].

The Analyzer of Space Plasmas and Energetic Atoms (ASPERA-3) [Barabash et al., 2006], the ion mass spectrometer aboard Mars Express, suggested a different view regarding ion precipitation than suggested by modeling. Hara et al. [2011] reported the first detection of precipitating heavy $\mathrm{O}^{+}$with energies around $1 \mathrm{keV}$ during a corotating interaction region (CIR) event and observed precipitating flux of the same order of magnitude as modeled by Chaufray et al. [2007]. However, ASPERA-3 only observed such an intensity of precipitating ion fluxes during CIR events, while Chaufray et al. [2007] simulated such an intensity for quiet solar wind conditions. Hara et al. [2013] published the first statistics on this heavy pickup ion precipitation based on one Martian year of ASPERA-3 measurements and observed that ion precipitation is usually organized by the convective electric 
field (in agreement with numerical simulations, Chaufray et al. [2007] and Li et al. [2011]). ASPERA-3 temporal and angular resolutions (192 s being needed to get a full angular $90^{\circ} \times 360^{\circ}$, energy and mass coverage) could explain this limited number of observations of precipitation.

In this paper, we present an analysis of the first 6 months of MAVEN science operation [Jakosky et al., 2015] observations of heavy pickup ion precipitation ( 700 MAVEN orbits). In section 2, we present the different instruments used for this study and their data sets. In section 3, an observation of pickup ion precipitation is described in detail and we reconstruct the average precipitating flux during the first 6 months of observations. Finally, we make a first estimate of the atmospheric escape rate that should result from the heavy ion precipitation at Mars.

\section{Instruments and Method}

In order to reconstruct the precipitating flux along the MAVEN trajectory, we used the two ion spectrometers of MAVEN, the Supra-Thermal And Thermal Ion Composition (STATIC; J. McFadden et al., The MAVEN Suprathermal and thermal Ion Composition (STATIC) instrument, submitted to Space Science Reviews, 2015) and the Solar Wind lon Analyzer (SWIA) [Halekas et al., 2013]. STATIC is an energy, mass, and angular ion spectrometer, operating in the range of $0.1 \mathrm{eV} / \mathrm{q}$ to $30 \mathrm{keV} / \mathrm{q}$ (energy resolution between 11 and 16\%), with a field of view (FOV) of $360^{\circ} \times 90^{\circ}$ (with $22.5^{\circ}$ resolution) and a mass range from 1 to 70 amu. STATIC has a mass resolution large enough to separate the main ion species: $\mathrm{O}_{2}{ }^{+}, \mathrm{CO}_{2}{ }^{+}$, and $\mathrm{O}^{+}$. For this study, we used essentially two products of STATIC measurements with $4 \mathrm{~s}$ time resolution: a product (corresponding to 32 bins in energy and 64 bins in mass) providing the mass and energy distributions of the ions measured by STATIC (without angular resolution) and a second product (corresponding to 16 bins in energy, 4 bins along the polar direction, and 16 bins in azimuth) providing the ion energy and angular distributions without the mass composition. STATIC has three different operational modes corresponding to three different energy ranges: Ram mode ( $0.1 \mathrm{eV}-50 \mathrm{eV})$, Conic mode $(0.1-500 \mathrm{eV})$, and Pickup mode ( $2.7 \mathrm{eV}-31,000 \mathrm{eV})$. Along a typical periapsis track, the STATIC mode changes from Pickup mode above 800-1000 km in altitude to Conic mode (above $200 \mathrm{~km}$ and below $1000 \mathrm{~km}$ ) then to Ram mode below 200-400 km in altitude. It should be noted that this sequence of modes is not systematically applied so that the respective altitude range might change significantly from one periapsis to another. Moreover, STATIC used mechanical and electrostatic attenuators to increase the dynamic range of the instrument. STATIC is mounted on the Articulated Payload Platform, which points STATIC's field of view into the Ram direction at periapsis (J. McFadden et al., submitted manuscript, 2015).

SWIA is an energy and angular ion spectrometer covering an energy range between $25 \mathrm{eV} / \mathrm{q}$ to $25 \mathrm{keV} / \mathrm{q}$ (with 48 logarithmically spaced energy steps) and a broad $360^{\circ} \times 90^{\circ}$ FOV. The energy resolution of SWIA is $14.5 \%$. The dynamic range of SWIA is increased due to the use of a mechanical attenuator. SWIA is positioned on the spacecraft in order to observe the direction of the solar wind most of the time but also to observe the nadir direction around periapsis. In this paper, we used the coarse survey product with an angular coverage of $360^{\circ} \times 90^{\circ}$, an angular resolution of $22.5^{\circ} \times 22.5^{\circ}$, and a temporal resolution of $4 \mathrm{~s}$ [Halekas et al., 2013].

Three other instruments of MAVEN could be also used to characterize the solar wind and solar flux conditions and to determine the presence of major crustal magnetic field structures: the EUV monitor, which is part of the Langmuir Probe and Waves instrument (LPW) [Andersson et al., 2015], the Solar Energetic Particle (SEP; D. E. Larson et al., The MAVEN Solar Energetic Particle Investigation, submitted to Space Science Reviews, 2015) instrument, and the magnetometer instrument (MAG) [Connerney et al. 2015]. The EUV monitor is designed to measure the solar irradiance in three bands from the soft X-ray to the extreme ultraviolet range $(0.1-7 \mathrm{~nm}, 17-22 \mathrm{~nm}$, and $121-122 \mathrm{~nm})$ with $1 \mathrm{~s}$ temporal resolution. The SEP instrument consists of two sensors measuring electrons and ions in the energy range 30-1000 keV and 30-12,000 keV, respectively, accommodated on two opposite corners of the S/C. The MAG instrument is designed to sample the magnetic field around Mars with two independent fluxgate magnetometers placed on extended solar array panels with an intrinsic rate of 32 vector samples/s. In this paper, we focus on one case study (postponing to other papers the study of the various drivers of this precipitation) and provide some first statistical elements regarding sputtering.

During the period from 11 November 2014 until 30 April 2015, these instruments performed nominal measurements of the ion fluxes around each periapsis. Excluding a few days during which instrument operations 
could not be performed, we are left with 150 days of useful measurements corresponding to around $\sim 1500$ inbound and outbound legs. In order to reconstruct the potential precipitating fluxes during each path, we selected a range in altitude between 200 and $350 \mathrm{~km}$. Ideally, precipitating flux should be calculated close to the Martian exobase [Yagi et al., 2012], that is, typically around $200 \mathrm{~km}$ in altitude. Below this theoretical limit, we assume that every downward moving ion should impact the atmospheric particles and be counted as a potential source of atmospheric sputtering. However, above the exobase, collisions between neutrals and ions are also rather probable (up to $50 \%$ through charge exchange above $200 \mathrm{~km}$ as discussed by Chaufray et al., 2007). Therefore, limiting the useful measurements to altitudes close to the exobase altitude might lead to an underestimate of the amount of energy precipitating into the atmosphere, part of it being already neutralized and partly thermalized. On the other hand, extending the sampling altitude to large distances from Mars might lead to an overestimate of the number of particles really impacting the atmosphere. We therefore selected an altitude of $350 \mathrm{~km}$ because, for most of the solar conditions at Mars, this altitude corresponds to a region below the induced magnetospheric boundary [Vignes et al., 2000; Edberg et al., 2008]. We then explicitly assumed that any ion measured between 350 and $200 \mathrm{~km}$ with a downward velocity oriented within a cone angle of $75^{\circ}$ centered along the radial direction will impact Mars' atmosphere. This assumption is probably valid for particles already significantly accelerated but less for low-energy particles. To move from 200 to $350 \mathrm{~km}$ in altitude, MAVEN needs typically $5 \mathrm{~min}$ during which about 75 individual full spatial and energy scans (with $4 \mathrm{~s}$ time resolution) were performed by STATIC and by SWIA. Within this period, we then summed, at each time step and for each energy bin, all the counts measured on anodes with their FOV within $75^{\circ}$ of the zenith direction. We postpone to future works a detailed discussion on the dependency of the precipitation intensity with respect to solar conditions when a significantly larger database will be available.

\section{Analysis}

In Figure 1, we show one example of precipitating heavy ion flux measured by STATIC and SWIA. These measurements were taken on 25 March 2015 near MAVEN's periapsis which occurred at 6:56:50 UTC (orbit 936). During this orbit, MAVEN was moving from a dayside periapsis to a nightside magnetotail apoapsis. We selected this case because measurements covering a few tens of eV up to a few tens of keV were simultaneously available from both STATIC and SWIA during the $200-350 \mathrm{~km}$ legs of the orbit and because a clear signature of heavy ion precipitation was observed. Indeed, most of the time, at low altitude, STATIC operates in Conic or Ram modes which do not cover the energy range most relevant for sputtering ( $1 \mathrm{keV})$ [Johnson et al., 2000]. During this orbit, MAVEN's periapsis was at $44.9^{\circ}$ east longitude and $16.8^{\circ}$ latitude in MSO (Mars Solar Orbital) coordinates and an altitude of $175 \mathrm{~km}$. MAVEN was within Mars' induced magnetosphere with the exception of a short period, before 06:19:20 UTC, where the spacecraft entered the undisturbed solar wind (Figures $1 \mathrm{a}$ and $1 \mathrm{~b}$ ). The average solar wind density was $5.6 \mathrm{~cm}^{-3}$, and the solar wind velocity was $438 \mathrm{~km} / \mathrm{s}$ (we estimated the solar wind conditions from SWIA plasma moments [Halekas et al., 2013] between 6:05UT and 6:18UT), while the interplanetary magnetic field and its variability measured by MAG was equal to $(-3.5 \pm 2.7,2.1 \pm 2.8,2.3 \pm 2.4) \mathrm{nT}$ in MSO coordinates. These solar wind observations and the magnetic measurements both suggest that the solar wind conditions were close to quiet with, however, a higher dynamic pressure than usual. During this particular periapsis, STATIC operated in pickup mode without attenuation of the ion flux (neither mechanical nor electrostatic).

During the low-altitude range of MAVEN orbit (06:49:00-07:04:00 UTC), STATIC changed its energy range to sample ions from $25 \mathrm{eV}$ to $31 \mathrm{keV}$ in order to avoid saturation, causing the solid white rectangle (i.e., no data) below $25 \mathrm{eV}$ in Figure $1 \mathrm{~b}$. As a consequence, fluxes in the mass-time spectrogram (Figure 1c) decrease significantly since ions below $25 \mathrm{eV}$ are not sampled. The energy-time spectrogram also displays some signatures of an energetic population up to $1 \mathrm{keV}$ (composed of $\mathrm{O}^{+}$and larger masses ions) just after 6:49:20. There is also good agreement between the measurements made by STATIC (Figure 1b) and SWIA (Figure 1a) within the common energy range because both FOVs were close to each other during that particular MAVEN periapsis pass. There is also a clear feature around $1 \mathrm{keV}$ below $200 \mathrm{~km}$ in altitude measured by both SWIA and STATIC which has been shown to be produced by solar wind protons double-charge exchanging with atmospheric neutrals [Halekas et al., 2015].

Figure 2 displays the observed average upward (dashed lines) and downward (solid lines) differential particle fluxes as measured by SWIA (red) and STATIC (blue) within an altitude range of $200-350 \mathrm{~km}$ on the inbound 

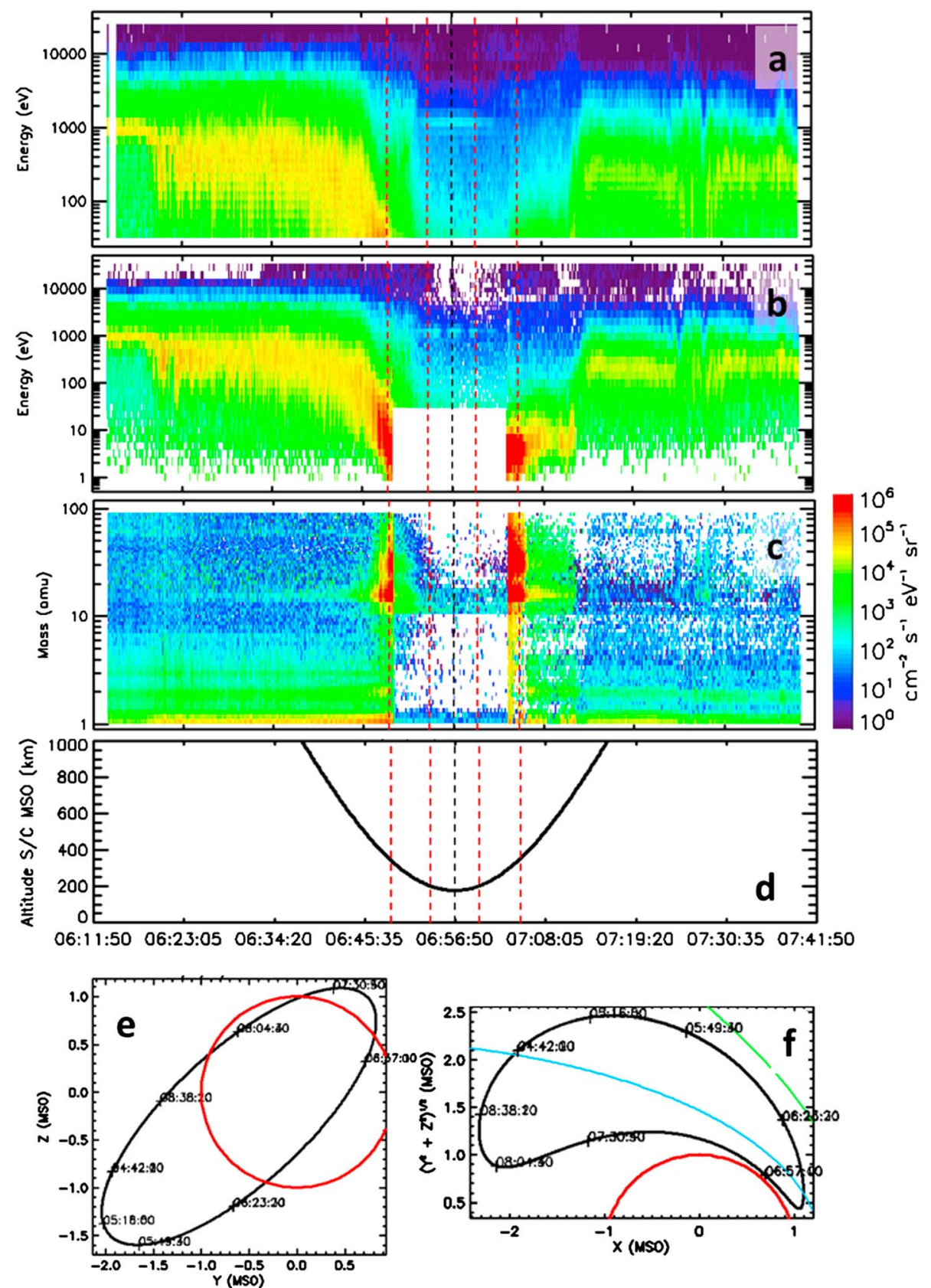

Figure 1. SWIA and STATIC measurements around MAVEN periapsis on 25 March 2015 at 6:56:50 UTC (the differential particle flux per anode multiplied by its FOV, summed over all anodes). At periapsis, MAVEN was at a MSO longitude of $44.9^{\circ}$, a latitude of $16.8^{\circ}$, and an altitude of $175 \mathrm{~km}$ (vertical dashed black line). The MSO right-handed coordinate frame is defined with the $X$ axis pointing from the center of Mars toward the Sun and the $Z$ axis pointing northward from the orbital plane of Mars. (a) SWIA energy-time spectrogram. (b) STATIC energy-time spectrogram. (c) STATIC mass-time spectrogram. The periods during which the precipitating ion flux is reconstructed are indicated by two pairs of vertical dashed red lines (altitude range 350-200 km). (d) Altitude of MAVEN. (e) Projected trajectory of MAVEN in a $Y, Z$ MSO plane. (f) Projected trajectory of MAVEN in an $X,\left(Y^{2}+Z^{2}\right)^{1 / 2}$ MSO plane. The blue curve and the green curve are the mean positions of the induced magnetosphere boundary and the bow shock, respectively, as inferred from Mars Global Surveyor [Vignes et al., 2000]. The red curve shows Mars. The Sun is to the right in Figure 1f. The white color corresponds to no measurement or too low fluxes in panels Figures 1a-1c.

leg for the same orbit. The STATIC profile below $25 \mathrm{eV}$ corresponds to the few measurements done below $350 \mathrm{~km}$ before and after the change of energy range as discussed above. As implied by Figure 1, there is overall a very good agreement between SWIA and STATIC for both the upward and downward fluxes during this time interval. The FOVs of both instruments were close and covering a large part of the cones defining both 


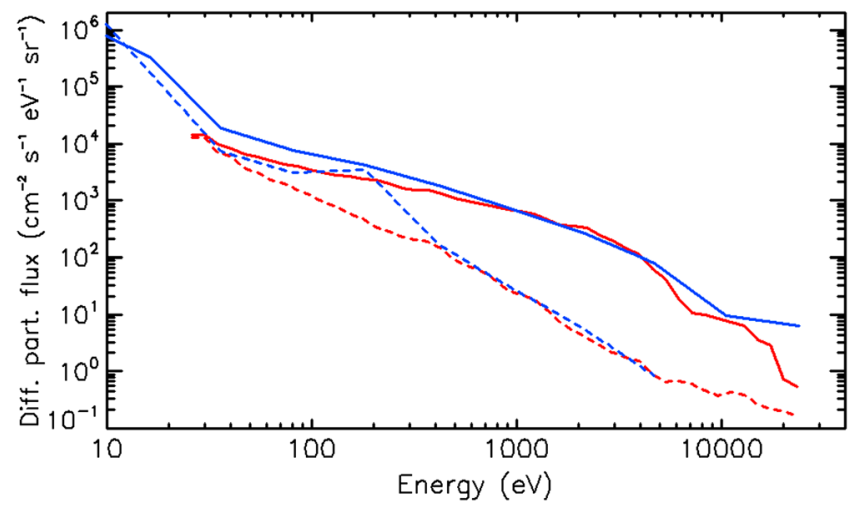

Figure 2. Reconstructed differential particle fluxes (all masses) measured by STATIC (binned over 16 energy intervals) and SWIA (binned over 48 energy intervals) between 200 and $350 \mathrm{~km}$ in altitude during the inbound path of MAVEN on 25 March 2015 (orbit 936). Blue lines are for STATIC measurements, whereas the red lines are for SWIA measurements. Dashed lines are for upward moving ions and solid lines for downward moving ions. The downward (upward) direction corresponds to all the anodes whose FOV is within $75^{\circ}$ of the local zenith (nadir) direction. $6 \times 10^{5}$ particles $/ \mathrm{cm}^{2} / \mathrm{s} / \mathrm{sr}, 20$ times larger than the upward flux and 3 to 4 times larger than the horizontal flux. As shown in Figure 1c, these fluxes are essentially composed of ions with mass larger than 10 to few tens of atomic mass units. During this time interval, the magnetic field was around $60 \mathrm{nT}$ (partly due to a moderate crustal magnetic field), the EUV flux measured by LPW/EUV was not available during that period and the SEP instruments measured ion and electron integral energy fluxes around $1300 \mathrm{eV} /\left(\mathrm{cm}^{2} \mathrm{~s} \mathrm{sr}\right)$ between 30 and $1000 \mathrm{keV}$ and $\sim 175 \mathrm{eV} /\left(\mathrm{cm}^{2} \mathrm{~s} \mathrm{sr}\right)$ between 30 and $300 \mathrm{keV}$, respectively. Therefore, these precipitating fluxes were obtained during relatively quiet solar conditions and most probably not significantly altered by the moderate crustal field intensity observed around periapsis.

Based on the first 6 months of MAVEN measurements, it is possible to build an average precipitating ion flux (mostly heavy ions) from both STATIC and SWIA data. However, because we focus on the observations by MAVEN at low altitude, this reconstruction is naturally dependent on MAVEN's periapsis position during this period. MAVEN's periapsis moved from high northern latitude $\left(60^{\circ} \mathrm{MSO}\right)$ around noon local time (midOctober 2014) into an eclipse period from the end of November 2014 to the end of February 2015, ending at northern latitude (10 $\mathrm{MSO}$ ) near noon local time at the end of April 2015 [see Jakosky et al., 2015, Figure 17]. Among the six months that have been analyzed, three months correspond to observations performed on nightside at midlatitude. As discussed in Lillis et al. [2015] and illustrated in Brain et al. [2015], we expect precipitation to occur mostly at high latitude on the dayside, driven by the upstream solar wind convective electric field, which is directed most of the time toward the north or the south depending on the sign of the IMF $B_{y}$ component in the MSO frame. Therefore, low latitudes and nightside regions are less favorable to observe the strongest expected precipitation.

Average precipitating ion fluxes (mostly heavy ions) are presented in Figure 3. For all periapsis passes from the first 6 months of observations (both dayside and nightside), downward (as defined above) fluxes measured in the altitude range $200-350 \mathrm{~km}$ are averaged to provide a single precipitating energy spectrum (Figure 3, top panel). Error bars on each instrument energy spectrum indicate the standard deviation calculated from all downward energy spectra with respect to the mean energy spectrum and therefore reflect the spatial and temporal variabilities of the precipitating ion fluxes. These fluxes are significantly larger than the detector background for both instruments. Differences between the two measured energy spectra can be explained by their different FOVs as mentioned in section 2. Indeed, on the 1500 individual passes through the $200-350 \mathrm{~km}$ altitude range, more than $90 \%$ of the observations made covered more than half of the cone angle centered on the zenith direction. STATIC and SWIA covered on average $66 \%$ and $61 \%$ of this cone angle, respectively. If we restrict the selection to anodes whose FOV is within $45^{\circ}$ from the zenith direction, then only $56 \%$ of STATIC observations cover more than $50 \%$ of this cone angle but $83 \%$ of SWIA observations 

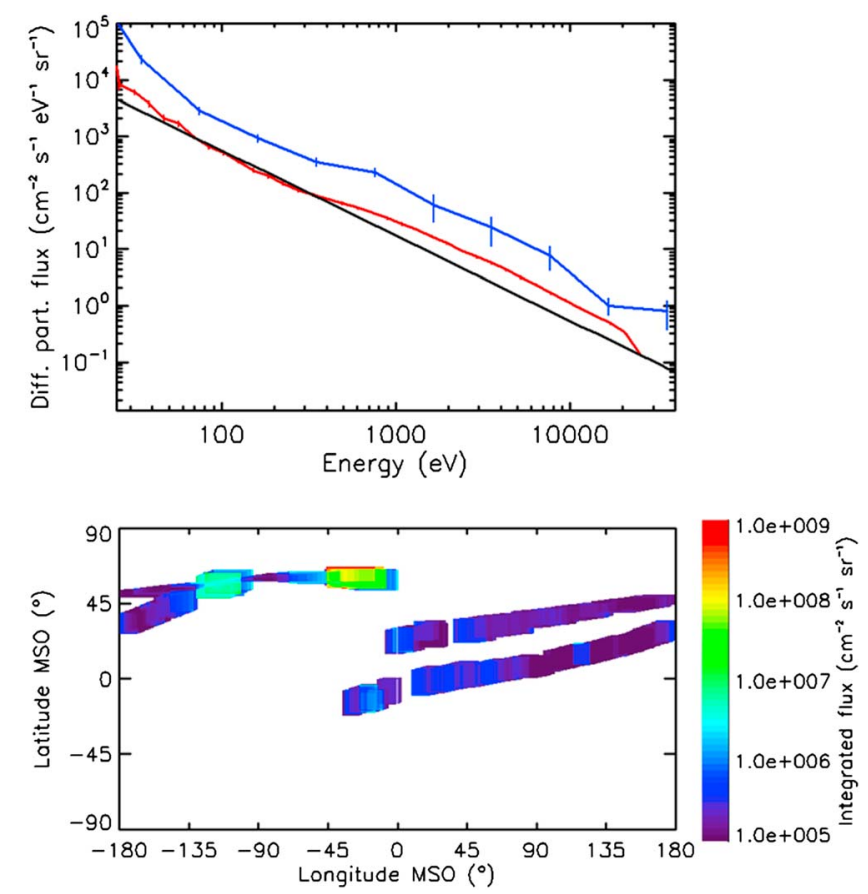

Figure 3. (top) Average downward ion energy spectrum in differential particle flux as measured by SWIA (red) and STATIC (blue) reconstructed from the measurements by SWIA and STATIC performed between 11 November 2014 and 30 April 2015. Around 1500 individual measurements between 200 and $350 \mathrm{~km}$ have been used to reconstruct those fluxes. We select only those measurements during which more than $50 \%$ of the nadir/zenith-centered $75^{\circ}$ cone angle was covered. Error bars on these two lines correspond to 1 sigma deviation calculated from all measured downward fluxes. The black curve shows the theoretical global average precipitating pickup $\mathrm{O}^{+}$energy spectrum calculated by Wang et al. [2015]. (bottom) Distribution of the average intensity of the integrated (from $25 \mathrm{eV}$ to $30 \mathrm{keV}$ ) energy distribution (in $\mathrm{cm}^{-2} \mathrm{~s}^{-1}$ ) with respect to the MSO longitude and latitude (binned over a $0.1^{\circ} \times 0.1^{\circ}$ grid). The subsolar point is at $\left(0^{\circ} \times 0^{\circ}\right)$. The colored regions correspond to the regions covered by MAVEN measurements between 200 and $350 \mathrm{~km}$ in altitude. metry with much less intense precipitation on the nightside (in agreement with Hara et al. [2013]). We also observed precipitation on the dayside at low latitudes but significantly smaller than at higher latitudes. All these features are consistent with the expected global organization of the precipitating flux at Mars [Lillis et al., 2015].

In order to estimate the sputtered escape rate associated with this precipitating flux, Wang et al. [2015] proposed a simple analytical relation based on a large set of simulations. Using the SWIA observations, we estimated the average integral precipitating flux during 6 months of observations within an energy range from $25 \mathrm{eV}$ to $30 \mathrm{keV}$ as being equal to $3 \times 10^{5} \mathrm{O}^{+} / \mathrm{cm}^{2} / \mathrm{s} / \mathrm{sr}$. Using Table 5 (coefficient $S_{3}$ and $C_{3}$ for O) and equation (14) of Wang et al. [2015], we then calculated a maximum sputtered escape flux of $6.3 \times 10^{5} \mathrm{O} / \mathrm{cm}^{2} / \mathrm{s}$ (or a rate of $1 \times 10^{24} \mathrm{O} / \mathrm{s}$ if averaged globally on a sphere of which radius corresponds to an altitude of $350 \mathrm{~km}$ ) on average during these last 6 months.

\section{Conclusion}

In this paper, we first show one clear example of ion precipitation of $\mathrm{O}^{+}$ions under quiet solar wind conditions (orbit 936), suggesting that precipitation is not as sporadic as suggested in Hara et al. [2011] and Diéval et al. [2013]. The first 6 months of observations of the two ion analyzers STATIC and SWIA are used to reconstruct the average precipitating flux of heavy ions and its distribution in MSO coordinates. 
Acknowledgments

F.L., R.M., and J.Y.C. are indebted to the program "Système Solaire" of CNES, the French space administration for its financial support on MAVEN. All data used in this paper are archived and available in the Planetary Data System Archive. R.L., S.C., J.L., J.H., T.H., D.L., F.E J.C., J. M., and B.J. were supported by the MAVEN project.
The global organization of the flux close to the exobase as well as the average energy distribution are in agreement with theoretical expectations. We therefore used the theoretical relations between integral precipitating energy flux and sputtered escaping flux [Wang et al., 2015] to propose a first estimate of Mars' atmospheric escape flux and rate induced by sputtering. According to this calculation, since the beginning of MAVEN science operations, $6.3 \times 10^{5} \mathrm{O} / \mathrm{cm}^{2} / \mathrm{s}$ (or $1 \times 10^{24} \mathrm{O} / \mathrm{s}$ ) escaped on average Mars' atmosphere due to sputtering.

\section{References}

Andersson, L., R. E. Ergun, and G. Delory (2015), The Langmuir probe and waves experiment for MAVEN, Space Sci. Rev., doi:10.1007/s11214015-0194-3.

Barabash, S., et al. (2006), The Analyzer of Space Plasmas and Energetic Atoms (ASPERA-3) for the Mars Express mission, Space Sci. Rev., 126(1-4), 113-164, doi:10.1007/s11214-006-9124-8.

Brain, D. A., et al. (2015), The spatial distribution of planetary ion fluxes near Mars observed by MAVEN, Geophys. Res. Lett., 42, doi:10.1002/ 2015 GL065293.

Chaufray, J. Y., R. Modolo, F. Leblanc, G. Chanteur, R. E. Johnson, and J. G. Luhmann (2007), Mars solar wind interaction: Formation of the Martian corona and atmospheric loss to space, J. Geophys. Res., 112, E09009, doi:10.1029/2007JE002915.

Connerney, J. E. P., J. Espley, P. Lawton, S. Murphy, J. Odom, R. Oliversen, and D. Sheppard (2015), The MAVEN Magnetic Field Investigation, Space Sci. Rev., doi:10.1007/s11214-015-0169-4.

Diéval, C., G. Stenberg, H. Nilsson, N. J. T. Edberg, and S. Barabash (2013), Reduced proton and alpha particle precipitations at Mars during solar wind pressure pulses: Mars Express results, J. Geophys. Res. Space Physics, 118, 3421-3429, doi:10.1002/jgra.50375.

Edberg, N. J. T., M. Lester, S. W. H. Cowley, and A. I. Eriksson (2008), Statistical analysis of the location of the Martian magnetic pileup boundary and bow shock and the influence of crustal magnetic fields, J. Geophys. Res., 113, A08206, doi:10.1029/2008JA013096.

Halekas, J. S., E. R. Taylor, G. Dalton, G. Johnson, D. W. Curtis, J. P. McFadden, D. L. Mitchell, R. P. Lin, and B. M. Jakosky (2013), The Solar Wind Ion Analyzer for MAVEN, Space Sci. Rev., doi:10.1007/s11214-013-0029-z.

Halekas, J. S., et al. (2015), MAVEN observations of solar wind hydrogen deposition in the atmosphere of Mars, Geophys. Res. Lett., 42, doi:10.1002/2015GL064693, in press.

Hara, T., K. Seki, Y. Futaana, M. Yamauchi, M. Yagi, Y. Matsumoto, M. Tokumaru, A. Fedorov, and S. Barabash (2011), Heavy-ion flux enhancement in the vicinity of the Martian ionosphere during CIR passage: Mars Express ASPERA-3 observations, J. Geophys. Res., 116, A02309, doi:10.1029/2010JA015778.

Hara, T., K. Seki, Y. Futaana, M. Yamauchi, S. Barabash, A. O. Fedorov, M. Yagi, and D. C. Delcourt (2013), Statistical properties of planetary heavy-ion precipitations toward the Martian ionosphere obtained from Mars Express, J. Geophys Res. Space Physics, 118, 5348-5357, doi:10.1002/jgra.50494.

Jakosky, B. M., et al. (2015), The Mars Atmosphere and Volatile Evolution (MAVEN) mission, Space Sci. Rev., doi:10.1007/s11214-015-0139-x.

Johnson, R. E., D. Schnellenberger, and M. C. Wong (2000), The sputtering of an oxygen thermosphere by energetic $\mathrm{O}^{+}$, J. Geophys. Res., 105, 1659-1670, doi:10.1029/1999JE001058.

Li, L., Y. Zhang, Y. Feng, X. Fang, and Y. Ma (2011), Oxygen ion precipitation in the Martian atmosphere and its relation with the crustal magnetic fields, J. Geophys. Res., 116, A08204, doi:10.1029/2010JA016249.

Lillis, R. J., et al. (2015), Characterizing atmospheric escape from Mars with MAVEN, today and in the ancient past, Space Sci. Rev., doi:10.1007/ s11214-015-0165-8.

Luhmann, J. G., R. E. Johnson, and M. H. G. Zhang (1992), Evolutionary impact of sputtering of the Martian atmosphere by O(+) pickup ions, Geophys. Res. Lett., 19, 2151-2154, doi:10.1029/92GL02485.

Luhmann, J., and J. U. Kozyra (1991), Dayside pickup oxygen ion precipitation at Venus and Mars-Spatial distributions, energy deposition and consequences, J. Geophys. Res., 96, 5457-5467, doi:10.1029/90JA01753.

Vignes, D., C. Mazelle, H. Rème, M. H. Acuña, J. E. P. Connerney, R. P. Lin, D. L. Mitchell, P. Cloutier, D. H. Crider, and N. F. Ness (2000), The solar wind interaction with Mars: Locations and shapes of the bow shock and the magnetic pile-up boundary from the observations of the MAG/ER Experiment onboard Mars Global Surveyor, Geophys. Res. Lett., 27, 1944-8007, doi:10.1029/1999GL010703.

Wang, Y.-C., J. G. Luhmann, F. Leblanc, X. Fang, R. E. Johnson, Y. Ma, W.-H. Ip, and L. Li (2014), Modeling of the O ${ }^{+}$pickup ion sputtering efficiency dependence on solar wind conditions for the Martian atmosphere, J. Geophys. Res. Planets, 119, 93-108, doi:10.1002/ 2013JE004413.

Wang, Y.-C., J. G. Luhmann, X. Fang, F. Leblanc, R. E. Johnson, Y. Ma, and W.-H. Ip (2015), Statistical studies on Mars atmospheric sputtering by precipitating pickup O+: Preparation for the MAVEN mission, J. Geophys. Res. Planets, 120, 34-50, doi:10.1002/2014JE004660.

Yagi, M., F. Leblanc, J. Y. Chaufray, F. Galindo-Gonzalez, and R. Modolo (2012), Mars exosphere: Thermal and non-thermal components: Seasonal and local variations, Icarus, 221(2), 682-693, doi:10.1016/j.icarus.2012.07.022. 